being felt two inches below the costal margin. On March 1st the cough had diminished, as also had the expectoration, but the character remained the same. The friction rub had disappeared. The temperature had remained normal throughout. Between March 18th and April 3rd the cough persisted and the expectoration was as before. The patient complained of pain on both sides of the chest, especially on movement. A nodular enlargement of the extremity of the eleventh rib on the right side was noticed which was very tender on pressure. There was similar tenderness on pressure over the eighth left rib in the midaxillary line. No nodule was detected. The spleen was as before, the abdomen was rather distended, and the edge of the liver was just felt. During this time the iron and arsenic pills were discontinued. On April 7th the patient felt rather faint in the morning and complained of nausea and general bodily pains; the anæmia had become noticeable again. In the evening he passed a black, tarry stool. The iron and arsenic were resumed and the patient was put back on the milk diet and ordered absolute rest in bed. On the 9th the melæna continued. Hæmatemesis (one pint) recurred after a stool (the patient being in bed). The pulse in the morning was 94 per minute and the temperature was $98.6^{\circ} \mathrm{F}$. The edge of the liver was felt half an inch below the costal margin. The spleen was as before. There was no glandular enlargement. The patient was ordered rectal feeding for eight days, to be followed by a milk diet; the arsenic and iron were continued. Melæna persisted till the 14th. The pulse had now become rapid, varying from 100 to 120 . The respirations were 24 per minute and the temperature was normal. On the 16th the differential count of white corpuscles was normal. The blood film showed no abnormal blood cells but, however, considerable poikilocytosis and polychromatophilic degeneration of Ehrlich. A distinct nodule was detected over the eighth left rib in the mid-axillary line. On the 18th the pulse was 104, the respirations were 24 , and the temperature was normal. The spleen was felt two inches below the costal margin. The abdomen was much distended and the patient complained of flatulence. The cough was still troublesome. Rhonchi were heard over both lungs scattered everywhere and there was much crepitation at both bases. The expectoration was white and frothy with occasional streaks of blood. By the 30th the edge of the liver was felt about one and a half inches below the costal margin. Two nodules of about the size of a small walnut could be distinctly made out over the anterior surface of the liver, which was rather tender on examination. The spleen was as before. The abdominal distension was now very considerable and there were signs of free fluid in the flanks. The cough was very troublesome. The anæmia was very profound but there was no trace of jaundice. The pulse was 110 and the temperature was $99^{\circ} 6^{\circ}$. The case now presented features suggestive of malignant disease, rapidly increasing weakness and emaciation, with anorexia, and nodular enlargement of the liver. The enlargement was more pronounced in the left lobe. There was also nodular thickening over certain ribs, probably of metastatic origin. No enlarged glands were to be detected anywhere. There were no symptoms by which one could localise the primary focus.

The further history of the case may be given shortly. The liver became enlarged and finally reached some four inches below the costal margin in the right nipple line, the edge of the liver being traced to the left across the epigastrium to the tenth rib; the nodules on its surface were easily apparent through the abdominal wall. The spleen retracted somewhat under the ribs but continued very firm and hard. The ascites was increased. The lung signs remained the same but the cough became very distressing. During the last four weeks of life there was irregular pyrexia varying between $99^{\circ}$ and $102^{\circ}$ and the pulse never fell below 108 per minute. The stools became very offensive, of a greenish colour, and contained much mucus, there being from two to three motions a day. Incontinence of fæces developed a fortnight before death, which occurred on June 23rd, during which time the patient was in a semi-comatuse condition.

Permission to examine the thorax and the abdomen after death was obtained, with the following result. Both lungs were found to be emphysematous and the pleura was adherent over the outer aspect of the lower lobes of both the right and the left lungs. In the left apex was found a small nodule of growth, of a soft, semi-fluid consistence, hæmorrbagic, and about one inch in diameter. A similar but larger nodule was found in the upper lobe of the right lung. The mediastinal glands were very much enlarged and on section showed infiltration with the same soft neoplastic material. The fifth and eighth ribs on the left side and the ninth and eleventh ribs on the right side showed metastatic nodules about one and a half inches in diameter, the rib in each case being fractured in the centre of the growth. The heart was enlarged slightly, with strong pericardial adhesion over almost its whole surface, the muscle being rather soft and pale. There was no valvular disease. The abdomen was considerably distended and a few pints of clear, reddishyellow fluid were removed from it. The liver was very much enlarged and irregular, the enlargement of the left lobe being very conspicuous. Over the anterior surface were scattered several projecting nodules of dark-red colour of about the size of a walnut and of soft consistence which on section were found to consist of hæmorrhagic, diffluent, semi-fluid material. On section through the whole organ numerous such nodules of varying size were brought to view and in each case the neoplastic deposit was in a disintegrated semi-fluid condition. There was no involvement of the gall-bladder. The spleen was uniformly enlarged (eight inches in its long diameter), very firm, and was bound by strong adhesions to the under surface of the diaphragm and the left aspect of the stomach, where the adhesions were of a cartilaginous consistence. The capsule was very much thickened and covered, especially on its posterior surface, with much lymph exudation which was partially organised. On section there was no sign of neoplasm but the organ was very fibrous throughout and engorged with blood. The stomach was rather smaller than normal. The mucous membrane showed very extensive, probably recent, ecchymosis over a large part of the fundus corresponding to the area of the stomach adherent to the spleen. Over the pyloric region posteriorly was much dark pigmentation, probably the result of old ecchymosis. There was no ulceration of the mucous membrane in either the stomach or the duodenum. The walls of the stomach were thin and the mucous membrane was rather atrophied. There was no affection of any of the abdominal lymphatic glands; the right inguinal glands were slightly enlarged and infiltrated with the soft neoplastic material. There was nothing abnormal to note concerning the intestines, the pancreas, or the suprarenals. The kidneys were pale, slightly enlarged, and rather fatty ; the capsule stripped easily. Owing to the very soft nature of the growth and the fact of its already being in a disintegrated condition microscopical examination was unsatisfactory. No definite conclusion as to the exact nature of the growth could be arrived at, though it was in all probability carcinomatous.

The above is a summary of the principal features of a case which is interesting both from the diagnostic and clinical point of view. It may be granted fairly, I think, that the malignant disease, wherever the primary focus lay, was not the primary cause of the illness, but that it supervened on splenic anæmia. Another point, which touches on some recent observations on the causation of malignant disease and its relation to arsenical administration, is the fact that the patient for some years previously had been treated with arsenic for psoriasis and with a very considerable amount of relief.

Grove Park, S.E.

\section{A CASE OF SUBACUTE INDURATIVE} PNEUMONIA.

By O. K. Williamson, M.A., M.D., B.C. Cantab, M.R.C.P. LOND.,

MEdICAL REgISTRAR TO THE MIDDLESEx HOSPITAL; PHYSICIAN TO OUT-PATIENTS, CITY OF LONDON HOSPITAL FOR DISEASES OF THE CHEST, VIOTORIA-PARK; AND

J. G. EMANUEL, M.D., B.S., B.Sc., M.R.O.P. LoND., PHYSTCIAN TO OUT-PATIENTS, QUEFN'S HOSPITAL, BIRMINGHAM; LATE RESIDENT MEDICAL OFFICER, CITY OF LONDON HOSPITAL FOR DISEASES OF THE CHEST, VICTORIA-PARK.

A GLRL, aged 17 years, was admitted into the City of London Hospital for Diseases of the Chest, Victoria-park, E., on Feb. 27th, 1900, under the care of Dr. T. Glover Lyon, complaining of pains in the right side of the chest, cough, expectoration, shortness of breath, and weakness. She had been ill for six weeks and the last four weeks of this time she had been in bed. Her previous health had 
been good, excepting that she had had scarlet fever seven years and measles six years previously. Six weeks before admission she complained of pain in the right shoulder which lasted for about four days and then disappeared. Ten days later, however, it reappeared and she was advised by her medical attendant to go to bed, and this she did, not on account of the gravity of her symptoms, however, but "because her doctor told her to." She remained at home in bed for four weeks and as she steadily lost ground she went to the hospital and was admitted. During these four weeks the pain in the right side of the chest had been constant and it had been aggravated by coughing and by taking deep inspirations. During this time she had also suffered from cough accompanied by expectoration. She had only noticed that her breath was short for one week and during this week she had had profuse night sweats. Two weeks prior to admission she had coughed up about half a pint of blood and had been expectorating stained sputum ever since. She had been getting paler, losing flesh, eating and sleeping badly, and getting gradually weaker during the four weeks she was in bed at home.

On admission the patient was pale, looked ill, and had recently lost much flesh. The pulse was 96 and the temperature was $98.6^{\circ} \mathrm{F}$. She had no dyspncea as she lay in bed. She had some cough and was expectorating blood-stained sputum. On examination of the chest the expansion of the right side was found to be impaired and that of the left side was exaggerated, The results of the examination of the right lung were as follows. Anteriorly, over the upper three spaces, the percussion note was unimpaired, the breath sounds were weak, expiration was prolonged and equal to inspiration, and both vocal resonance and vocal fremitus were diminished. Below the third space there was dulness which became continuous with the hepatic dulness, the breath sounds were weak and bronchial, vocal resonance and vocal fremitus were absent, and there was whispering pectoriloquy. Posteriorly, in the supraspinous fossa, the percussion note was unimpaired, the breath sounds were a little weak, and expiration was prolonged and equal to inspiration. The vocal resonance and the vocal fremitus were normal. Below the spine of the scapula and in the axilla the percussion note was dull, the breath snunds were weak and amphoric, the vocal resonance and the vocal fremitus were diminished, and there were ægophony at the angle of the scapula and whispering pectoriloquy all over the base. The breath sounds were faintly audible down to the very base. There were no adventitious sounds throughout the chest. In the left lung, anteriorly and posteriorly, the percussion note was resonant, the breath sounds were exaggerated, the vocal resonance and the vocal fremitus were normal, and the entry at the base was good. The cardiac impulse was in the fourth space in the vertical nipple line and the heart sounds were clear at all the cardiac areas. Examination of the abdomen revealed nothing abnormal, the liver and the spleen were not palpable, and the urine was normal.

The case ran a downward course from the beginning. The patient died three months after her admission to hospital. During these three months the temperature continued irregularly intermittent, the evening temperature varying between $101^{\circ}$ and $102 \cdot 5^{\circ}$, while the morning temperature fell to $98^{\circ}$ or $99^{\circ}$. The pulse-rate was always above 100 per minute and during the last month it ranged from 120 to 130 per minute. During the latter six weeks of this time diarrhoea was a prominent symptom, the patient having three or four loose motions daily.

With the exception of the free hæmoptysis which occurred two weeks before the patient went to the hospital there was not any free hæmorrhage from the lungs, but during the whole of the time that she was under observation at the hospital the sputum was stained with blood. The sputum was again and again examined for tubercle bacilli but with a negative result. The cough continued all the time and was constantly accompanied by blood-stained expectoration. During the first four weeks the expectoration was small in amount, averaging about one ounce per day, muco-purulent, and not offensive, but one day (March 30th) the patient suddenly brought up, after a paroxysm of coughing, a large quantity of very offensive, purulent, grey-coloured sputum. From this time onwards she continued to cough up daily large quantities of very offensive, often blood-stained sputum averaging six ounces a day; on 14 days the sputum measured over ten ounces and on two occasions $14 \frac{1}{2}$ ounces were measured.
Owing to the belief that the patient was suffering from an empyema communicating with the right lung or from an abscess in the base of the lung, the right chest was explored with a needle on five occasions but without finding the source of the pus. Some time in April the sputum was noticed to be reddish-brown and closely to simulate socalled "liver pus," and an abscess of the liver communicating with the lung was suggested. On May 7th a sixth exploration of the right chest in the seventh space midway between the right vertical nipple line and the right edge of the sternum discovered exceedingly offensive, strawyellow, watery pus. A portion of a rib was accordingly resected, a quantity of this highly offensive straw-coloured watery pus was evacuated, and the cavity was drained. No communication could be made out at the operation between this empyema cavity and the lung, nor, indeed, was such a communication anticipated, since the pus that was being expectorated, though it resembled the evacuated pus in point of its great offensiveness, differed in its other characteristics, for one was straw-coloured and watery and the other was reddish-brown and much thicker. As the patient did not improve after the draining of this empyema, but was fast losing ground, and as the "liver pus" continued to be expectorated in large quantities it was resolved to explore the liver and accordingly an exploratory laparotomy was performed on May 16th. At the operation the liver was found to be enlarged but in other respects it was quite healthy. The patient died from broncho-pneumonia of the hitherto apparently normal left lung on the third day, the laparotomy wound being bealthy.

Necropsy.-At the post-mortem examination the body was found to be wasted. The abdominal wound was healthy, there being recent adhesions around it. The wound in the thorax was also healthy and here the two layers of the pleura were seen to be separated, forming a cavity the length of which from above downwards was about four or five inches. Everywhere else the visceral and parietal layers of the right pleura were adberent, the adhesions being very firm and the pleura being thickened throughout except at the apex of the upper lobe. It was impossible to separate the lung from the chest wall without tearing its substance, and the diaphragm, the diaphragmatic pleura, and the lung were firmly incorporated with one another. The visceral pleura at the site of the interlobar fissures was covered with yellow lymph. The left visceral pleura showed some recent inflammation on the outer surface of the lung rather below its middle but there were neither fluid nor adhesions on this side. There were a few fibrous adhesions between the parietal pericardium and the sternum with some excess of clear fluid in the pericardial cavity but no signs of recent inflammation. The peritoneum was normal. The right lung weighed $28 \frac{1}{2}$ ounces and the left weighed $20 \frac{1}{2}$ ounces. The right lung was markedly diminished in size and was noncrepitant. On section the upper lobe was pale and practically airless ; it was homogeneous in appearance and its consistence was markedly increased. In the lower lobe, extending from about one and a half inches below the apex to the base and nearer the posterior than the anterior surface, was a large, irregularly shaped cavity, the walls of which contained fibrous tissue. In colour the walls were in some parts green, in other parts red, and the cavity contained some foulsmelling fluid and particles of necrosed lung tissue. The cavity was torn open on removing the lung. The rest of the lower lobe and the middle lobe were similar to the upper, but not of uniform colour and more friable, showing in places areas of thick pus of the size of a millet seed. The left lung was emphysematous as regards the upper lobe, and the lower lobe for the most part was non-crepitant. On section there was found to be some oedema of the upper part. The lower part, especially the posterior part of the lower lobe, exhibited light-coloured patches of broncho-pneumonia of the size of a pea, coalescing to form a solid mass which broke down easily and which was granular on the surface. There were on this side also some areas of thick pus of the size of a millet seed. This lung showed no obvious fibrosis. There was no dilatation of the bronchi in either lung. The heart weighed eight ounces, its cavities contained some antemortem clot, and its muscle was rather more friable than normal. The aorta and pulmonary artery were healthy. The liver weighed 70 ounces. It was very large, especially as regards the right lobe. Over a considerable area it was firmly adherent to the diaphragm. On section it was pale and markedly friable. The spleen weighed seven and a half ounces and was found on section to be firmer and more brittle than normal. The kidneys each weighed six and a 
half ounces and were on section found to be rather more friable than normal. The suprarenal capsules and the pancreas were normal, as was also the large intestine. The small intestine, especially the ileum, showed marked congestion of the mucous membrane. In the right broad ligament was a cyst of the size of a large cherry. There was nowhere any evidence of tuberculosis.

Mioroscopical examination.-Sections of the lungs stained with carbol-fuchsin and methylene blue failed to reveal the presence of tubercle bacilli. Sections were made from the centre of the upper lobe of the right lung and from the left lung. The left lung showed distinct fibrosis, although there were no naked-eye signs of this, but the process was seen to be much less advanced on this side than on the right. In the sections from both lungs the alveoli were in places seen to contain a mass of coarsely granular material with many nuclei, sometimes connected with the wall of the alveolus by a band of connective tissue. In places this central plug was evidently becoming converted into fibrous tissue. The walls of the alveoli were also in places seen to be thickened. In other parts the cavities of the alveoli were completely obliterated by fibrous tissue, indicating a more advanced stage of the process, this being observed more especially in the right lung. Besides the above changes there was evidence of fibrous thickening of the interstitial tissue of the right lung.

The rarity of subacute indurative pnenmonia is our reason for publishing in detail this case. Whether this rarity is more apparent than real it is difficult to say, for it is conceivable that many actual examples of this condition occur but do not come to the post-mortem table and so are returned as cases of pulmonary tuberculosis, bronchiectasis, abscess of lungs, gangrene of lung, or as unresolved pneumonia. Although we have to confess that we did not diagnose this case during life, but thought we were dealing either with an empyema which we could not localise and which had burst into the lung or else with an abscess of the lung of unexplained origin, nevertheless, looking back on the case with the light thrown on it by the post mortem examination we find that many of the points that clinically distinguish this subacute form of pneumonia from other conditions were present in a typical manner. The onset, for example, though sudden, was not the abrupt onset of acute pneumonia. There was no initial rigor. The onset was marked by sharp pain in the side which was sufficiently severe for the patient to call in a medical man, but for which she did not take to bed for a full fortnight, and then only "because the doctor told her to" and not because she was feeling too ill to be up and about. This brings out another feature of the case-viz., the fact that the illness immediately following the onset had not the gravity of an acute pneumonia, by which a patient is generally compelled to take to bed from the first When the patient first came under our observation, six weeks it will be remembered after the beginning of her illness, she presented the physical signs of a cavity at the right base, and we then inclined to a diagnosis of basal pulmonary tuberculosis This suggestion was strengthened by the occurrence of hæmoptysis early in the case and by the sputum being constantly blood-stained later. Repeated examinations of the sputum failed, however, to reveal tubercle bacilli, and we consequently put pulmonary tuberculosis out of court.

The sudden change which occurred in the character and quantity of the sputum on March 30th (ten weeks after the beginning of the illness) made us, from that day on, strongly incline towards the diagnosis of a deep-seated empyema bursting into the lung. For before this day the sputum was small in amount, muco-purulent, and not offensive. And then it suddenly became abundant, purulent, and foetid in character. Subsequent events proved this change to be due to the emptying of a gangrenous cavity in the lower lobe of the right lung.

The progressively downward course of the case from the very first, and the fatal termination in gangrene of the lung after an illness of months only (the entire duration of the case was four and a half months), are characteristic. The diarrhoea of the last six weeks is also not an infrequent symptorn. The small empyema that was evacuated on May 7th (only 11 days before death) was in our opinion secondary to the septic condition of the lung, and must be looked upon as a complication of the case, and one of not very common occurrence, since, as a rule, in these cases the pleural cavity becomes obliterated at any early period by fibrous adhesions.
The post-mortem examination revealed a large gangrenous cavity in the right lung, with secondary broncho-pneumonia; and the naked-eye changes generally agreed with those characteristic of subacute indurative pneumonia. This was confirmed by microscopical examination of sections of the lungs, the left showing the process in an early stage. This examination showed: (1) the absence of tubercle bacilli; and (2) the fact of the conversion of the contents of the alveoli into fibrous plugs.

We wish to thank Dr. Glover Lyon for permission to publish this case.

Bibliography.-J. K. Fowler : The Diseases of the Lungs (Fowler and Godlee), p. 237. Percy Kidd: The LanCET, April 5th, 1890, p. 740 Wagner: Deutsches Archiv für Klinische Medicine, Band xxxiii. Heitler : Wiener Medicinische Wochenschrift, 1884 and 1886. Talma:
Zeitschrift fuir Klinische Medicin. Band x. Hanau : Ibid., Band. xii. Pal : Fortschritte der Medicin, 1888.

\section{ON THE OCCURRENCE AND SIGNIFI- CANCE OF CUTANEOUS HYPER- ALGESTA IN APPENDICITIS.}

BY JAM ES SHERREN, F.R.C.S. ENG., ASSISTANT SURGEON TO THE LONDON HOSPITAL AND SURGEON TO THE POPLAR HOSPITAL FOR ACCIDENTS.

VERY little attention seems to have been paid by surgeons to the subject of cutaneous tenderness in appendicitis and in the more recent text-books of surgery and monographs on appendicitis no mention is made of it. Head ${ }^{1}$ and Mackenzie ${ }^{2}$ independently in 1892 first drew attention to the subject of cutaneous hyperalgesia in visceral disease. In these articles no special attention is paid to its occurrence in appendicitis, but Head mentions that in affections of the intestine down to the upper part of the rectum superficial tenderness may appear in the ninth, tenth, and eleventh dorsal segmental areas. In the same paper Head quotes a case showing the absence of this hyperalgesia in perforative appendicitis and he has taught for some years (although I cannot find definite reference to it in any of his published works) that this superficial tenderness, if present, disappears on perforation of the appendix. In the third edition of Quain's "Dictionary of Medicine" Head in his article on Pain has given a later account of the subject but has made no special reference to the appendix. In the ten years intervening between Head's two papers 1 can find in the literature no mention of the subject.

In December, 1902, Müller read a paper at the Freie Vereinigung der Chirurgen Berlins on Head's Areas in Surgical Abdominal Disease. ${ }^{3}$ In this paper he mentions "that he has found hyperalgesia in early cases of appendicitis with tolerable frequency, this hyperalgesia usually appearing in the neighbourhood of McBurney's point." Nothing further is said on the subject in this paper. Mackenzie ${ }^{4}$ in a paper on the Nature of the Symptoms in Appendicitis mentions the occurrence of this cutaneous hyperalgesia but does not discuss its significance or the form of the disease in which it is present. Mansell Moullin ${ }^{5}$ in a clinical lecture on the Significance of Pain and Tenderness in Cases of Inflammation of the Appendix mentions the presence of hyperæsthesia in most cases of appendicitis and also draws attention to the fact that "its disappearance suggests very strongly that the appendix has become gangrenous."

In order to discover the frequency with which this sign is present in appendicitis and to test the teaching that it disappears on perforation of the appendix $I$ undertook this research, hoping to find an additional guide to operation in appendicitis. For this purpose during the time that I was surgical registrar at the London Hospital I examined all the cases of appendicitis that were admitted to the hospital as far as circumstances would permit. But I have based my observations entirely upon cases in which the notes were taken by myself and in which the diagnosis of appendicitis

I Brain, vol. xvi 2 Medical Chronicle, 1892

3 Centralblat für Chirurgie, No. 2, 1903.

Brit. Med. Jour., July 11 th, 1903

5 THE Lancer, August 22nd, 1903, p. 514 